\title{
Trading Off Space for Passes in Graph Streaming Problems
}

\author{
CAMIL DEMETRESCU, \\ IRENE FINOCCHI \\ and ANDREA RIBICHINI \\ Università di Roma "La Sapienza", Roma, Italy
}

Data stream processing has recently received increasing attention as a computational paradigm for dealing with massive data sets. Surprisingly, no algorithm with both sublinear space and passes is known for natural graph problems in classical read-only streaming. Motivated by technological factors of modern storage systems, some authors have recently started to investigate the computational power of less restrictive models where writing streams is allowed. In this paper, we show that the use of intermediate temporary streams is powerful enough to provide effective spacepasses tradeoffs for natural graph problems. In particular, for any space restriction of $s$ bits, we show that single-source shortest paths in directed graphs with small positive integer edge weights can be solved in $O\left(\left(n \log ^{3 / 2} n\right) / \sqrt{s}\right)$ passes. The result can be generalized to deal with multiple sources within the same bounds. This is the first known streaming algorithm for shortest paths in directed graphs. For undirected connectivity, we devise an $O((n \log n) / s)$ passes algorithm. Both problems require $\Omega(n / s)$ passes under the restrictions we consider. We also show that the model where intermediate temporary streams are allowed can be strictly more powerful than classical streaming for some problems, while maintaining all of its hardness for others.

Categories and Subject Descriptors: F.2.3 [Analysis of Algorithms and Problem Complexity]: Tradeoffs between Complexity Measures; G.2.2 [Discrete Mathematics]: Graph Theory

General Terms: Algorithms

Additional Key Words and Phrases: Data streaming, Graph connectivity, Shortest paths

\section{INTRODUCTION}

The typical data size of a wide range of applications in computational sciences can easily reach the order of Terabytes or even Petabytes. In all such applications managing massive data sets, using secondary and tertiary storage devices is a practical

\footnotetext{
C. Demetrescu, Dipartimento di Informatica e Sistemistica, Università di Roma "La Sapienza", Roma, Italy. Email: demetres@dis.uniroma1.it. URL: http://www.dis.uniroma1.it/ demetres.

I. Finocchi, Dipartimento di Informatica, Università di Roma "La Sapienza", Roma, Italy. Email: finocchi@di.uniroma1.it. URL: http://www.dsi.uniroma1.it/ finocchi.

A. Ribichini, Dipartimento di Informatica e Sistemistica, Università di Roma "La Sapienza", Roma, Italy. Email: ribichini@dis.uniroma1.it. URL: http://www.dis.uniroma1.it/ ibichini.

Work supported in part by the Italian MIUR Project "MAINSTREAM: Algorithms for massive information structures and data streams". A preliminary version of this paper appears in the Proceedings of the 17th Annual ACM-SIAM Symposium on Discrete Algorithms (SODA'06). Permission to make digital/hard copy of all or part of this material without fee for personal or classroom use provided that the copies are not made or distributed for profit or commercial advantage, the ACM copyright/server notice, the title of the publication, and its date appear, and notice is given that copying is by permission of the ACM, Inc. To copy otherwise, to republish, to post on servers, or to redistribute to lists requires prior specific permission and/or a fee.

(c) $20 \mathrm{ACM} 0004-5411 / 20 / 0100-\$ 5.00$
} 
and economical way to store and move data: such large and slow external memories, however, are best optimized for sequential access, and thus naturally produce huge streams of data that need to be processed in a small number of sequential passes. Typical examples include data access to database systems [Golab and Ozsu 2003] and analysis of Internet archives stored on tape [Henzinger et al. 1999]. Information naturally occurs in the form of huge data streams also in applications that monitor in real-time network traffic, on-line auctions, transaction logs such as Web usage logs, telephone call records or automated bank machine operations [Gilbert et al. 2001; Golab and Ozsu 2003; Sullivan and Heybey 1998]. Among the computational models that have been proposed to deal with massive data sets, data stream processing has therefore received an ever increasing attention in the last few years.

In the classical data stream model [Henzinger et al. 1999; Munro and Paterson 1980; Muthukrishnan 2003], input data can be accessed sequentially in the form of a data stream, and need to be processed using a working memory that is small compared to the length of the stream. The main parameters of the model are the number $p$ of sequential passes over the data and the size $s$ of the working memory (in bits): throughout this paper, we will refer to the class of problems solvable within $p$ passes using working memory $s$ as $\operatorname{Stream}(p, s)$. A typical additional parameter is the per-item processing time, which should also be kept small. Despite the heavy restrictions of the Stream model, major success has been achieved for several data sketching and statistics problems, where $O(1)$ passes and polylogarithmic working space have been proven to be enough to find approximate solutions (see, e.g., [Alon et al. 1999; Feigenbaum et al. 2002; Gilbert et al. 2002] and the bibliographies in [Babcock et al. 2002; Muthukrishnan 2003]). On the other hand, many other problems seem to be far from being solved within similar bounds, including most classical graph problems. Relevant examples are graph connectivity and shortest paths, for which linear lower bounds on $p \times s$ are known [Henzinger et al. 1999]. Some recent papers show that several graph problems can be solved with one or few passes in the semi-streaming model [Feigenbaum et al. 2004; 2005; McGregor 2005] where the working memory size is $O(n \cdot \operatorname{polylog} n)$ for an input graph with $n$ vertices: in other words, akin to semi-external memory models [Abello et al. 2002; Vitter 2001] there is enough space to store vertices, but not edges of the graph. While $O(n \cdot$ polylog $n)$ space seems to be a "sweet spot" for streaming graph problems [Muthukrishnan 2003], a natural question already posed in [Henzinger et al. 1999; Munro and Paterson 1980] is whether we can reduce the space usage at the price of increasing the number of passes. Surprisingly, to the best of our knowledge no algorithms with both sublinear space and passes are known for natural graph problems in the Stream model. Finding effective space-passes tradeoffs in this context appears therefore to be a challenging research direction for both its theoretical and practical implications. Consider for instance the problem of processing a very large graph stored on a file in secondary memory. On a standard computing platform with 1 GB of available main memory, a tradeoff algorithm that runs in $p=(n \log n) / s$ passes ${ }^{1}$ can process a graph with 4 billion vertices and 6 billion edges stored in a $50 \mathrm{~GB}$ file in less than 16 passes. Using a RAID disk with $100 \mathrm{MB} / \mathrm{sec}$ sequential access rate, this would take roughly 2.5 hours. With

\footnotetext{
${ }^{1}$ Throughout this paper, we assume that all logarithms are to the base 2 .
} 
a streaming algorithm that requires $s \geq n \log n$ bits without being able to trade space for passes, we would simply not be able to solve the problem at hand (even with infinite time) unless 16 GB of main memory are available.

Motivated by technological factors, some authors have recently started to investigate the computational power of less restrictive streaming models. Today's computing platforms are equipped with large and inexpensive disks highly optimized for sequential read/write access to data, and among the primitives that can efficiently access data in a non-local fashion, sorting is perhaps the most optimized and well understood. These considerations have led Aggarwal et al. [Aggarwal et al. 2004] to introduce the "streaming and sorting" model, denoted here as StreamSort. This model extends Stream in two ways: the ability to write intermediate temporary streams and the ability to reorder them at each pass for free. A StreamSort algorithm alternates streaming and sorting passes: a streaming pass, while reading data from the input stream and processing them in the working memory, produces items that are sequentially appended to an output stream; a sorting pass consists of reordering the input stream according to some (global) partial order and producing the sorted stream as output. Streams are pipelined in such a way that the output stream produced during pass $i$ is used as input stream at pass $(i+1)$. As shown in [Aggarwal et al. 2004; Ruhl 2003], the combined use of intermediate temporary streams and of a sorting primitive yields enough power to solve efficiently (within polylogarithmic passes and polylogarithmic memory) a variety of problems, including graph connectivity, minimum spanning tree, and geometrical problems. It remains however an open question whether problems such as shortest paths (and even breadth first search) can be solved efficiently in this more powerful model.

Since random accesses in external memory are significantly slower than sequential passes, Grohe et al. have also proposed an abstract model that captures the essence of external memory and stream processing [Grohe et al. 2005]. This model restricts the size of the main memory and the number of random accesses to external memory, but does not restrict sequential reads. Similarly to StreamSort, the model admits the usage of external memory for storing intermediate results. Lower bounds for sorting the input data and for other decision problems in this model have been proved in [Grohe et al. 2006; Grohe et al. 2005; Grohe and Schweikardt 2005]. Very recently, these results have been extended to 2-sided error randomized algorithms [Beame et al. 2007].

$\triangleright$ Our contributions. In this paper we show that the StreamSort model can yield interesting results even without using sorting passes at all: by just using intermediate temporary streams, we provide effective space-passes tradeoffs for natural graph problems. Namely, if we denote by $W$-Stream this more restrictive model without a sorting primitive [Ruhl 2003], we show that for any space restriction of $s$ bits:

- Undirected connectivity can be solved in $W$-Stream by a deterministic algorithm in $O((n \log n) / s)$ passes. By adapting classical communication complexity arguments previously used in the Stream model, we can prove an $\Omega(n / s)$ lower bound on the number of passes for connectivity also in $W$-Stream. Our algorithm is thus optimal up to a logarithmic factor.

- Single-source shortest paths in directed graphs with positive integer edge weights up to $C$ can be solved in $W$-Stream by a randomized Monte Carlo algorithm 
in $O\left(\left(C n \log ^{3 / 2} n\right) / \sqrt{s}\right)$ passes. The result can be generalized to deal with $\rho$. $\sqrt{s / \log n}$ sources within the same bounds for any $\rho \in(0,1)$. This is the first known algorithm for shortest paths on directed graphs in a streaming model. We remark that previous results on distances in streaming models are based on the computation of graph spanners, and these yield approximate distances in undirected graphs only [Feigenbaum et al. 2005]. We also note that the lower bound for connectivity implies an $\Omega(n / s)$ lower bound on the number of passes also for single-source (and thus multiple-sources) shortest paths.

We remark that for these problems we have exactly the same lower bounds on $p \times s$ in both Stream and in W-Stream. The only known upper bounds in Stream assume $s=\Theta(n \log n)$. On the other hand, our $W$-Stream algorithms adapt to the available working memory, yielding a full range of possible space/passes tradeoffs. This motivates us to conclude the paper with some observations related to the computational power of $W$-Stream.

A first natural question is whether the use of temporary streams always makes $W$-Stream more powerful than Stream in a multi-pass setting. In this paper, we give a negative answer, by providing examples of problems that are as hard in $W$-Stream as in Stream for a given space restriction, regardless of the number of passes. One such example is the classical element distinctness problem, where the challenge is to determine whether a given input stream contains any duplicates. This hardness result can be proved using classical tools from communication complexity. We remark that this kind of arguments can be applied to both Stream and W-Stream, but not to StreamSort.

Intuitively, however, the use of intermediate temporary storage should make $W$ Stream more powerful than Stream, at least for some problems. We exemplify two different ways in which this is indeed the case. We first observe that, from a classical space complexity perspective, intermediate streams can be thought of as part of the algorithm's working memory. So clearly there can be problems impossible to solve in Stream with a given space restriction, but solvable in $W$-Stream in a finite number of passes. The recognition of context-free languages is one prominent example. As a second observation, we note that in $W$-Stream the size of intermediate streams can vary from pass to pass, while in Stream the same input stream is read at each pass. Counting the total number of processed stream items, rather than the number of passes, may therefore be a more accurate measure for comparing algorithms in the two models. Based on this observation, we show that there are problems for which the number of processed items in $W$-Stream can be asymptotically smaller than in Stream.

$\triangleright$ Notation. Throughout this paper, we will refer to the class of problems solvable in $W$-Stream within $p$ passes using a working memory of $s$ bits as $W$-Stream $(p, s)$. Similarly, $\operatorname{Stream}(p, s)$ will denote the class of problems solvable in Stream within $p$ passes and space $s$. When dealing with algorithms for graph problems, we will assume that the input graph is given as an adjacency stream [Bar-Yossef et al. 2002], i.e., as a stream $\Sigma$ of edges in arbitrary order, with $|\Sigma|=m$.

$\triangleright$ Organization of the paper. The remainder of this paper is organized as follows. In Section 2 we study the undirected connectivity problem, describing an almost optimal $W$-Stream deterministic algorithm. Section 3 addresses the shortest paths 

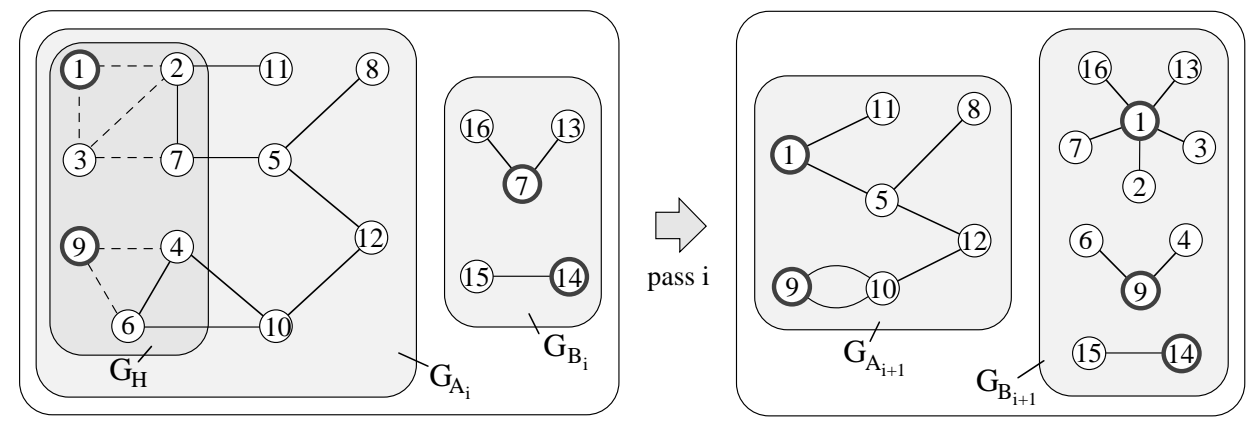

Fig. 1. Example of the effects of one pass of the connectivity algorithm

problem, presenting a randomized algorithm to find distances from a given set of sources to all the other vertices of a weighted directed graph. Section 4 discusses some aspects of the computational power of the $W$-Stream model. Concluding remarks are given in Section 5.

\section{UNDIRECTED GRAPH CONNECTIVITY}

In this section we show that using intermediate temporary streams we can achieve the first effective space/passes tradeoffs for a fundamental graph problem, namely the undirected connectivity problem (UCON) which, given an undirected graph $G=$ $(V, E)$ with $n$ vertices and $m$ edges, asks whether $G$ is connected.

By a classical communication complexity argument based on a reduction from the bit-vector disjointness problem (see [Henzinger et al. 1999]), UCON requires $\Omega(n / s$ ) passes in Stream when the space restriction is $s$, i.e., $U C O N \notin \operatorname{Stream}(o(n / s), s)$. The same communication complexity argument can be adapted to $W$-Stream, as we will discuss in Section 4, yielding the following theorem:

TheOREM 2.1. In W-Stream, UCON requires $p=\Omega(n / s)$ passes with a space restriction $s$.

$\triangleright$ Algorithm. We now describe a deterministic algorithm that solves the more general problem of finding the connected components of $G$ using $p=O((n \log n) / s)$ passes and space $s$ in the $W$-Stream model.

Given an undirected graph $G=(V, E)$, let $C(G)=\left(V, E^{\prime}\right)$ be the undirected graph on the same vertex set such that $(u, v) \in E^{\prime}$ if and only if $v$ is the representative vertex of the connected component of $G$ that contains $u$. We note that $C(G)$ represents explicitly the connected components of $G$ as stars around component representatives. If $L$ is a list of edges, we denote by $G_{L}=\left(V_{L}, L\right)$ the graph induced by edges in $L$. Thus, $G_{\Sigma}=G$.

The algorithm works as follows. Each intermediate stream $\Sigma_{i}$ produced by the algorithm is divided into two consecutive parts $A_{i}$ and $B_{i}$ such that $G_{B_{i}}$ is a collection of stars, and $G_{A_{i}} \cup G_{B_{i}}$ has the same connected components as $G$. At the beginning, $A_{0}=\Sigma$ and $B_{0}=\emptyset$, and thus $G_{A_{0}}=G$ and $G_{B_{0}}=\emptyset$. At the end, $G_{A_{p}}=\emptyset$ and $G_{B_{p}}=C(G)$ is the desired result. The generic pass $i$ of the algorithm works in four phases: 
(1) Read a prefix $H$ of edges from $A_{i}$ and store in main memory $M$ each newly encountered vertex until either $M$ gets full, or all edges of $A_{i}$ have been read. Let $G_{H}=\left(V_{H}, H\right) \subseteq G_{A_{i}}$ be the graph induced by the edges in the prefix $H$ of $A_{i}$ read in this phase. As edges are streamed in, also form in $M$ the connected components of $G_{H}$, e.g., by building a spanning forest. No output items are produced during this phase.

(2) Read all remaining edges from $A_{i}$ (if any). Let $c(v)$ be the representative vertex of the connected component of $G_{H}$ that contains $v$, if $v \in V_{H}$, and let $c(v)=v$ otherwise. For each input item $(u, v)$ read from $A_{i}$ such that $c(u) \neq c(v)$, write $(c(u), c(v))$ as output item to $A_{i+1}$.

(3) Read all edges from $B_{i}$. For each input edge $(u, v)$ read from $B_{i}$, write $(u, c(v))$ as output edge to $B_{i+1}$.

(4) No edges remain to be read from input stream. For each vertex $v$ in $V_{H}$ that does not appear in $A_{i+1}$, write $(v, c(v))$ as output edge to $B_{i+1}$.

The algorithm repeats the generic pass described above until $A_{i}$ gets empty. We note that phase 1 is a memory loading phase, which stores $V_{H}$ in main memory along with a sparse certificate of connectivity of $G_{H}$ (e.g., a spanning forest). Phase 2 produces an output graph $G_{A_{i+1}}$ obtained from $G_{A_{i}}$ by contracting each connected component of $G_{H}$ into its representative vertex. Vertices that are in $G_{A_{i}}$, but disappear from $G_{A_{i+1}}$ due to the contraction are put in $G_{B_{i+1}}$ by connecting them to their component representatives in $G_{H}$. These representatives may be later replaced by newer representatives in successive executions of phase 3 so as to maintain the invariant that $G_{B_{i}}$ is a collection of stars. The example of Figure 1 illustrates the effects of one pass of the algorithm.

$\triangleright$ Analysis. To prove the correctness of the algorithm, it suffices to check that the following invariant is maintained at each pass.

InVARIANT 2.2. For each $i \in\{0, \ldots, p\}, G_{B_{i}}$ is a collection of stars, and $G_{A_{i}} \cup$ $G_{B_{i}}$ has the same connected components as $G$.

Proof. We prove our claim by induction on the number of passes performed by the algorithm. The base for $i=0$ is straightforward, since $G_{A_{i}}=G$ and $G_{B_{i}}=\emptyset$. We assume by inductive hypothesis that the invariant holds at pass $i$, and we show that it also holds at pass $(i+1)$. First, observe that $G_{B_{i+1}}$ is obtained in phases 3 and 4 as union of stars from $G_{B_{i}}$ and stars that represent the connected components of $G_{H}$. If the stars produced in the two phases are not disjoint, the union may not be a collection of stars. For this reason, if a star in $G_{B_{i}}$ intersects a component of $G_{H}$, its center is replaced in phase 3 by its representative in $G_{H}$. Thus, $G_{B_{i+1}}$ is a collection of stars. To prove that $G_{A_{i+1}} \cup G_{B_{i+1}}$ has the same connected components as $G$, observe that each connected component of $G_{H} \subseteq G_{A_{i}} \subseteq G_{A_{i}} \cup G_{B_{i}}$ is replaced by a star in $G_{A_{i+1}} \cup G_{B_{i+1}}$, and thus connectivity information is maintained.

Assuming that the main memory $M$ has a size of $s$ bits, we now show that the algorithm terminates in at most $p=O((n \log n) / s)$ passes.

ThEOREM 2.3. In W-Stream, UCON can be solved with $p=O((n \log n) / s)$ passes when the space restriction is $s$. 
Proof. Without loss of generality, we assume that the input graph $G$ contains no self-loops. Indeed, self loops can be easily removed with a preprocessing phase that takes $O(n / s)$ passes. Notice that during pass $i$, all vertices of $V_{H}$ that are not representatives of connected components of $G_{H}$ disappear from $G_{A_{i+1}}$ (phase 2). Since $G_{H}$ is induced by a set of edges, each connected component of $G_{H}$ contains at least two vertices. Thus, there are at least $\left|V_{H}\right| / 2$ vertices in $G_{A_{i}}$ that disappear from $G_{A_{i+1}}$, so in at most $p \leq 2 n /\left|V_{H}\right|$ passes $G_{A_{p}}$ gets empty. Since phase 1 fills memory $M$ with vertices and a spanning forest of $G_{H}$ until it gets full, and storing each vertex label requires $\log n$ bits of space, then $\left|V_{H}\right|=\Theta(s / \log n)$. This implies that $p=O((n \log n) / s)$.

The crucial point in the analysis is the choice of $G_{H}$, which is the largest graph induced by a prefix of the stream such that a sparse certificate of its connected components fits in $s$ bits of memory. If $G_{H}$ is dense, we may contract at each pass a number of edges much larger than $s$. This makes the number of passes proportional to the number of nodes of the graph, instead of the number of edges.

\section{SHORTEST PATHS}

Let $G=(V, E, w)$ be an edge-weighted directed graph with $n$ vertices and $m$ edges. In the single-source shortest paths problem (SSSP), we wish to find distances from a given source $t$ to all the other vertices in $G$. A more general version of the problem is the multiple-sources shortest paths problem (MSSP), where the goal is to find distances from a given set of sources to all the other vertices in $G$. In this section, we assume that each edge $(u, v) \in E$ is represented in the input stream $\Sigma$ as a triple $\left(u, v, w_{u v}\right)$, where $w_{u v}$ is the weight of the edge. In the following, we assume that each vertex label and each edge weight can be represented with $\log n$ bits. We say that the weight of a path is the sum of the weights of its edges. The distance dist $x y$ between two vertices $x$ and $y$ of the graph is the weight of a minimum weight path connecting them.

We first observe that, since UCON can be reduced to SSSP, then the lower bound for UCON given in Theorem 2.1 also holds for SSSP (and thus for MSSP):

THEOREM 3.1. In W-Stream (and thus in Stream), SSSP requires $p=\Omega(n / s)$ passes when the space restriction is $s$.

This implies that, if we want to achieve sublinear space $s=o(n)$, then $p=\omega(1)$ passes are required. One may wonder whether $p=O(1)$ passes would be enough to solve the problem using $s=O(n)$ space. Unfortunately, as showed by Feigenbaum et al. in [Feigenbaum et al. 2005] a higher lower bound can be proven in the Stream model: the lower bound implies that finding vertices up to distance $d=O(1)$ from a given source in less than $d$ passes requires $\Omega\left(n^{1+1 / 2 d}\right)$ space. Since $p$ is constant and $W$-Stream can be simulated in Stream at the price of increasing the size of the working memory by a factor of $p$ (see [Ruhl 2003]), it is not difficult to see that this result also holds in $W$-Stream. This confirms that space efficient algorithms for SSSP in both Stream and W-Stream always require multiple passes.

We also remark that finding efficient streaming algorithms for the simpler problem of breadth-first traversal of a graph has been posed as an open problem even in the StreamSort model [Aggarwal et al. 2004]. 
In the remainder of this section, we devise the first algorithm for single-source shortest paths in directed graphs in a streaming model. In particular, we prove the following theorem:

ThEOREM 3.2. In W-Stream, MSSP from $\rho \cdot \sqrt{s / \log n}$ sources, for any $\rho \in$ $(0,1)$, can be solved with $p=O\left(\left(C \cdot n \cdot \log ^{3 / 2} n\right) / \sqrt{s}\right)$ passes in directed graphs with positive integer edge weights up to $C$ under a space restriction of $s$ bits. Distances produced by the algorithm are correct with probability at least $1-1 / n^{\beta}$ for any positive constant $\beta$. The size of each intermediate stream is $O(m+n \cdot \sqrt{s / \log n})$.

Notice that, for $C=o\left(\sqrt{s} / \log ^{3 / 2} n\right)$ we can get both $p$ and $s$ sublinear in $n$.

$\triangleright$ Overview of the algorithm. A typical issue in streaming settings where edges are given in arbitrary order is that following a path seems to require in the worst case as many passes as its length. Finding long paths may therefore require lots of passes. To overcome this difficulty, we argue that, if we were able to find long paths as the concatenation of short paths built "in parallel" within the same passes, this would result in a substantial reduction of the worst-case number of passes required to follow a path of arbitrary length. Similarly to previous algorithms for path problems in parallel and dynamic settings (see, e.g., [Henzinger and King 1995; Ullman and Yannakakis 1991]), the main idea of our algorithm is to perform many short searches from a random subset of vertices of the graph "in parallel". This yields short distances in the graph. To find longer distances, the algorithm stitches together short paths. Using a probabilistic argument, we can prove that distances obtained in this way are correct with high probability.

\subsection{Finding distances up to $\ell$}

Let $A=\left\{c_{1}, c_{2}, \ldots, c_{|A|}\right\}$ be a subset of vertices of the graph and let $\ell>0$ be an integer parameter. As a first ingredient for solving SSSP in $W$-Stream, we describe a procedure shortDist $(A, \ell)$ that finds the distances from each source $c_{j} \in A$ to all other vertices that are up to distance $\ell$ from $c_{j}$ in $p=O\left(\frac{n|A| \log n}{s}+\ell\right)$ passes in a graph with positive integer edge weights.

Our procedure is a multi-source, bounded depth, streamed implementation of Dijkstra's algorithm [Cormen et al. 2001], where the "priority queue" is maintained implicitly on intermediate streams. Let $\left\{\gamma_{1}, \gamma_{2}, \cdots, \gamma_{q}\right\}$ be a partition of the input stream $\Sigma_{0}=\Sigma$ into the minimum number $q$ of sub-sequences $\gamma_{i}$ such that:

-all edges in a sub-sequence $\gamma_{i}$ share the same end vertex $y_{i}$, i.e.,

$$
\gamma_{i}=\left(a, y_{i}, w_{a y_{i}}\right)\left(b, y_{i}, w_{b y_{i}}\right) \cdots\left(z, y_{i}, w_{z y_{i}}\right)
$$

- the concatenation of sub-sequences $\gamma_{i}$ yields $\Sigma_{0}$, i.e.,

$$
\Sigma_{0}=\gamma_{1} \gamma_{2} \cdots \gamma_{q}
$$

Notice that, if the edges in the input stream $\Sigma$ were ordered by their end vertex, there would exist a unique sub-sequence $\gamma_{i}$ per vertex. In general, the same end vertex may be shared by more than one sub-sequence, i.e., it may be $y_{a}=y_{b}$ with $a \neq b$. 
Each intermediate stream $\Sigma_{h}$, with $h>0$, created by the algorithm has the form

$$
\Sigma_{h}=\gamma_{1} \delta_{1} \gamma_{2} \delta_{2} \cdots \gamma_{q} \delta_{q}
$$

where

$$
\delta_{i}=\left(d_{i 1}, f_{i 1}\right)\left(d_{i 2}, f_{i 2}\right) \cdots\left(d_{i|A|}, f_{i|A|}\right) .
$$

For each pair $\left(d_{i j}, f_{i j}\right) \in \delta_{i}, d_{i j}$ is an upper bound to the distance dist $_{c_{j} y_{i}}$ from $c_{j} \in A$ to $y_{i}$ and flag $f_{i j}$ is true if and only if $y_{i}$ is settled w.r.t. $c_{j}$, i.e., dist $\operatorname{dis}_{c_{j} y_{i}}$ has been correctly determined by the algorithm.

In a preliminary pass, the algorithm lets $f_{i j}=$ false for each $i$ and $j$; it also lets $d_{i j}=0$ if $c_{j}=y_{i}$, and $d_{i j}=+\infty$ otherwise. The goal of successive passes is to progressively decrease each $d_{i j}$ to the weight of a minimum weight path from $c_{j}$ to $y_{i}$ that goes through one of the edges in $\gamma_{i}$.

The core loop of algorithm shortDist alternates extraction and relaxation passes. During an extraction pass, the algorithm loads in main memory, for each $c_{j} \in A$, a pool $P_{c_{j}}$ of at most $k=s /(|A| \cdot \log n)$ vertices $v$ together with their exact distance $d i s t_{c_{j} v}$ from $c_{j}$ (after the first extraction pass, each pool $P_{c_{j}}$ includes only vertex $c_{j}$ and $\left.d_{i s t_{c_{j} c_{j}}}=0\right)$. During a relaxation pass, the algorithm improves the distance upper bounds $d_{i j}$ using edges in $\gamma_{i}$ that emanate from $P_{c_{j}}$. In more details:

-Extraction pass. Let $d_{j}(v)=\min _{i: v=y_{i}}\left\{d_{i j}\right\}$ be the priority of $v$ w.r.t. $c_{j}$. For each $c_{j} \in A$, load in $P_{c_{j}}$ up to $k$ unsettled vertices with the same minimum priority w.r.t. $c_{j}$, if it does not exceed $\ell$. For each vertex $v$ in $P_{c_{j}}$, it holds $\operatorname{dist}_{c_{j} v}=d_{j}(v)$. When all $P_{c_{j}}$ 's get empty, the algorithm halts.

-Relaxation pass. For each $i=1,2, \ldots, q$, decrease each $d_{i j}$ in the output $\delta_{i}$ to the weight of a minimum weight path from $c_{j}$ to $y_{i}$ that goes through one of the edges in $\gamma_{i}$ that emanate from $P_{c_{j}}$. Also, make vertices in each $P_{c_{j}}$ settled w.r.t. $c_{j}$ by letting $f_{i j} \leftarrow$ true in the output stream for each $y_{i} \in P_{c_{j}}$.

We remark that all the vertices that are in each pool $P_{c_{j}}$ at the end of an extraction pass have exactly the same distance from $c_{j}$. All these vertices would be extracted from the priority queue in consecutive iterations of a classical implementation of Dijkstra's algorithm with source $c_{j}$. When the algorithm is over, for each $c_{j}$ and each vertex $v$ that is settled w.r.t. $c_{j}$, the distance $\operatorname{dist}_{c_{j} v}$ is implicitly encoded in the output stream as $\min _{i: v=y_{i}}\left\{d_{i j}\right\}$ and can be easily made explicit with a simple post-processing in $O((n \log n) / s)$ passes.

$\triangleright$ Analysis. We now discuss the number of passes required by algorithm shortDist.

LEMma 3.3. Algorithm shortDist $(A, \ell)$ runs in $p=O\left(\frac{n|A| \log n}{s}+\ell\right)$ passes using s bits of working memory and intermediate streams of size ${ }^{s}(m \cdot|A|)$.

Proof. The algorithm keeps in the working memory up to $k=s /(|A| \cdot \log n)$ vertices in each of the $|A|$ pools $P_{c_{j}}$. Since storing each vertex label requires $\log n$ bits, the algorithm uses at most $k \cdot|A| \cdot \log n=s$ bits of main memory. The bound on the size of intermediate streams follows from the fact that each of them contains $m+q \cdot|A|$ items and $q$ can be as high as $m$ in the worst case.

To bound the number of passes, consider the vertex $c_{j} \in A$ such that $P_{c_{j}}$ is the last pool to get empty. Let $P_{1}, P_{2}, \cdots, P_{t}$, be the content of pool $P_{c_{j}}$ after successive 
extraction passes. We say that $P_{i}$ is full if it contains exactly $k$ vertices, and it is incomplete otherwise. Notice that, since each vertex appears in at most one $P_{i}$, there can be at most $(n / k)$ full $P_{i}$ 's. We now bound the number of incomplete $P_{i}$ 's. Note that in each set $P_{i}$, all vertices have the same distance from $c_{j}$. Denote this distance by $d\left(P_{i}\right)$. Set $P_{i}$ can be incomplete only if $d\left(P_{i}\right)<d\left(P_{i+1}\right)$, or $i=t$. Since $d\left(P_{t}\right) \leq \ell$ and edge weights are positive integers, there can be at most $\ell$ passes $i$ such that $d\left(P_{i}\right)<d\left(P_{i+1}\right)$, and thus at most $\ell P_{i}$ 's can be incomplete. Hence, the total number $t$ of $P_{i}$ 's cannot exceed $(n / k+\ell)$. Since the algorithm generates a new $P_{i}$ every two passes in the core loop, then it performs a total number of $p=O(t)=O(n / k+\ell)=O\left(\frac{n|A| \log n}{s}+\ell\right)$ passes.

Notice that for $A=\{t\}$ algorithm shortDist solves SSSP up to distance $\ell=$ $O((n \log n) / s)$ from a given source $t$ in $O((n \log n) / s)$ passes. By Theorem 3.1, this bound is optimal in $W$-Stream up to a log factor.

$\triangleright$ Reducing the size of intermediate streams. In this section we show how to reduce the size of intermediate streams to $O(m+n \cdot|A|)$. The main idea is to preprocess the input stream so as to reduce the number $q$ of groups $\gamma_{i}$ before starting the shortDist algorithm. To this aim, we simply partition the input stream $\Sigma$ into $\max \{1, m /(n \cdot|A|)\}$ subsequences of size $\leq n \cdot|A|$ each, and we reorder edges $\left(x, y, w_{x y}\right)$ in each subsequence by end vertex $y$. This can be done in $O((n \cdot|A| \cdot \log n) / s)$ passes by using a $W$-Stream variant of the sorting algorithm described in [Munro and Paterson 1980].

The preprocessing can thus be performed within the same asymptotic number of passes as shortDist. Notice that the number of groups $\gamma_{i}$ in each reordered subsequence cannot be larger than $n$. Hence, the total number $q$ of groups $\gamma_{i}$ in the whole preprocessed stream given as input to shortDist will not exceed $n \cdot \max \{1, m /(n \cdot|A|)\}=\max \{n, m /|A|\}$. The size of each intermediate stream in shortDist will therefore be $m+q \cdot|A| \leq m+\max \{n \cdot|A|, m\}=O(m+n \cdot|A|)$ as claimed.

\subsection{Finding all distances from a given source}

We now describe an algorithm $\operatorname{sssp}(G, t)$ that solves $\operatorname{SSSP}$ with source $t$ in a graph $G=(V, E, w)$ with $n$ vertices and positive integer edge weights up to $C$ in $O\left(\left(C n \log ^{3 / 2} n\right) / \sqrt{s}\right)$ passes, assuming a space restriction of $s$ bits in the $W$ Stream model. Algorithm $\operatorname{sssp}(G, t)$ works as follows:

(1) Pick a subset $A \subseteq V$ of $\sqrt{s / \log n}$ vertices, including source $t$. All vertices but $t$ are chosen uniformly at random.

(2) Find distances up to $\ell=\left(\alpha C n \log ^{3 / 2} n\right) / \sqrt{s}$ in $G$ from each of the vertices in $A$, where $\alpha$ is any constant $>1$.

(3) Build a weighted graph $G^{*}=\left(A, E^{*}, w^{*}\right)$ on vertex set $A$ such that there is an edge $\left(c_{1}, c_{2}\right) \in E^{*}$ with weight $w_{c_{1} c_{2}}^{*}=\operatorname{dist}_{c_{1} c_{2}}$ if and only if $\operatorname{dist}_{c_{1} c_{2}} \leq \ell$.

(4) Compute distances dist $t_{c c}^{*}$ from $t \in A$ to all other vertices $c \in A$ in $G^{*}$.

(5) For each vertex $v \in V$ whose distance from $t$ has not been determined in step 2 being higher than $\ell$, compute it as dist $t_{t v}=\min _{c \in A}\left\{d i s t_{t c}^{*}+d i s t_{c v}\right\}$. 
Before describing a $W$-Stream implementation of sssp, we prove that each distance larger than $\ell$ computed by the algorithm is correct with high probability, assuming that distances up to $\ell$ computed in step 2 are correct.

Lemma 3.4. Each distance dist $t_{t v}>\ell$ computed by algorithm $\mathrm{sssp}$ is correct with probability at least $1-1 / n^{\alpha-1}$.

Proof. If $d i s t_{t v}>\ell$, then it is obtained in step 5 as $d i s t_{t v}=\min _{c \in A}\left\{d i s t_{t c}^{*}+\right.$ $\left.d i s t_{c v}\right\}$. Since edge weights of $G$ are $\leq C$, then any shortest path from $t$ to $v$ in $G$ will necessarily contain at least $r=\ell / C$ edges. Let $\pi_{t v}$ be any shortest path from $t$ to $v$. Adapting to our setting a well known sampling theorem from [Greene and Knuth 1982], we now show that, with high probability, every subpath of $\pi_{t v}$ with $r$ vertices contains at least a vertex from $A$. Consider one of those subpaths, and let $Q$ be the probability that it does not contain vertices of $A$. Since a vertex of $G$ belongs to $A$ with probability $|A| / n$, then:

$$
Q=\left(1-\frac{|A|}{n}\right)^{r}<2^{-\frac{|A| r}{n}}=\frac{1}{n^{\alpha}}
$$

Since there can be at most $n / r \leq n$ disjoint subpaths of $\pi_{t v}$ with $r$ vertices, then the probability that each of them contains a vertex of $A$ is at least $1-Q$. $n>1-1 / n^{\alpha-1}$. This implies that, with probability at least $1-1 / n^{\alpha-1}, \pi_{t v}$ can be broken into the concatenation of subpaths of at most $r$ vertices of the form $\pi_{c_{i} c_{j}}$, with $c_{i}, c_{j} \in A$, plus one final subpath of the form $\pi_{c^{*} v}$, where $c^{*}$ minimizes $\min _{c \in A}\left\{d i s t_{t c}^{*}+d i s t_{c v}\right\}$. Since w.h.p. each $\pi_{c_{i} c_{j}}$ is a shortest path with weight at most $\ell$, then it corresponds to an edge $\left(c_{i}, c_{j}\right) \in E^{*}$. Thus, w.h.p. the value $d i s t_{t c^{*}}^{*}$ computed in step 4 is the correct distance from $t$ to $c^{*}$. To conclude the proof, observe that $d i s t_{c^{*} v}$ has also weight at most $\ell$ w.h.p., and thus it has been correctly determined in step 2 . Thus, $d_{i s t_{t v}}=d i s t_{t c^{*}}^{*}+d i s t_{c^{*} v}$ with probability at least $1-1 / n^{\alpha-1}$.

$\triangleright$ Implementation. In this section we sketch how steps $1-5$ of algorithm sssp can be implemented in $W$-Stream:

(1) As $|A|=\sqrt{s / \log n}$, then vertices of $A$ can be sampled and maintained in main memory.

(2) To find distances up to $\ell$ from each vertex in $A$, we can just run algorithm shortDist $(A, \ell)$ described earlier in this section. The algorithm stores distances on the output stream.

(3) Graph $G^{*}$ can be stored in main memory, since it requires no more than $|A|^{2} \log n=s$ bits. To build it, we can just make one pass and build an $|A| \times|A|$ weight matrix $w^{*}$ such that for each $c_{j}, c \in A w_{c_{j} c}^{*}=\min _{i: c=y_{i}}\left\{d_{i j}\right\}$.

(4) Distances $d i s t_{t c}^{*}$ can be computed by running any internal-memory single-source shortest paths algorithm on $G^{*}$ with source $t$, and can be stored in main memory using $O(|A| / \log n)=O\left(\sqrt{s} \log ^{3 / 2} n\right)$ bits of space.

(5) Compute final distances for $s / \log n$ vertices $K \subseteq V$ at a time. For each $K$, compute in one pass dist $t_{t v}=\min _{i, j: v=y_{i}}\left\{d i s t_{t c_{j}}^{*}+d_{i j}\right\}$ for each $v \in K$. At the end of the pass, flush computed distances to the output stream in any desired format. 
$\triangleright$ Analysis. We now discuss the time and space requirements of algorithm sssp.

LEMma 3.5. Algorithm $\operatorname{sssp}(G, t)$ computes, correctly with high probability, the distances from $t$ to all nodes in $G$ within $O\left(\left(C n \log ^{3 / 2} n\right) / \sqrt{s}\right)$ passes, using $s$ bits of main memory and intermediate streams of size $O(m+n \cdot \sqrt{s / \log n})$ in the W-Stream model.

Proof. Steps 1 and 4 are entirely performed in main memory, and thus require no streaming passes. Step 3 requires one pass and step 5 takes $O((n \log n) / s)$ passes. The entire procedure is dominated by the number of passes of algorithm shortDist in step 2, which is $O\left(\frac{n|A| \log n}{s}+\ell\right)=O\left(\frac{C n \log ^{3 / 2} n}{\sqrt{s}}\right)$ by Lemma 3.3. The size of intermediate streams also follows from Lemma 3.3 and from the preprocessing technique described thereafter. The largest data structure maintained by the algorithm in main memory is matrix $w^{*}$ created in step 3, which requires $s$ bits. The claim on the correctness of the computed distances follows from Lemma 3.4

\subsection{Dealing with multiple sources}

The algorithm described in Section 3.2 can be extended to deal with $\rho \cdot \sqrt{s / \log n}$ sources within the same asymptotic bounds, for any $\rho \in(0,1)$. Of the $\sqrt{s / \log n}$ vertices loaded into set $A$ in step 1 , let only a $(1-\rho)$ fraction of these be chosen uniformly at random, while the remaining vertices are taken to be sources for the shortest paths problem. In step 2, we find distances up to $\ell /(1-\rho)$. Using algorithm shortDist $(A, \ell /(1-\rho))$ this will take $O\left(\frac{C n \log ^{3} / 2 n}{\sqrt{s}}\right)$ passes. Notice that, since only a $(1-\rho)$ fraction of $A$ is chosen at random, algorithm shortDist needs to find paths of length up to $\ell /(1-\rho)$. In view of Lemma 3.4, this is crucial for maintaining correctness with high probability. Step 3 of the algorithm remains unchanged, while in step 4 the distances from each source to all other vertices in main memory can be computed, by running any internal-memory single-source shortest paths algorithm for each source vertex. The results can be stored in internal memory. The final distances can be computed by repeating step 5 of the original algorithm once for every source. Since there are $\rho \cdot \sqrt{s / \log n}$ sources, this will take $O\left(\sqrt{\frac{s}{\log n}} \cdot \frac{n \log n}{s}\right)=O\left(\frac{n \log ^{1 / 2} n}{\sqrt{s}}\right)$ passes. Therefore the number of passes of the entire algorithm is determined by the asymptotic performance of the procedure shortDist, and this is the same as in the single-source case. We call the algoritm described in this section mssp and we summarize its bounds in the following lemma.

Lemma 3.6. Algorithm mssp requires $O\left(\left(C n \log ^{3 / 2} n\right) / \sqrt{s}\right)$ passes, using $s$ bits of main memory and intermediate streams of size $O(m+n \cdot \sqrt{s / \log n})$ in the $\mathrm{W}$ Stream model. With high probability, all computed distances are correct.

\section{SEPARATION AND HARDNESS RESULTS}

The algorithms presented in Sections 2 and 3 show that the ability to write intermediate streams makes it possible to obtain, at least for some problems, a full range of possible space/passes tradeoffs, whereas the only known upper bounds in Stream assume $s=\Theta(n \log n)$. These results naturally raise the question of whether $W$-Stream is more powerful than Stream. In this section we therefore 
study some aspects related to the computational power of $W$-Stream. We first exemplify problems in W-Stream that are impossible to solve in Stream for a given space restriction and problems that require a smaller number of processed items in $W$-Stream than in Stream. Note that in $W$-Stream the size of intermediate streams can vary from pass to pass, while in Stream the same input stream is read at each pass: counting the total number of processed stream items, rather than the number of passes, may therefore be a more accurate measure for comparing algorithms in the two models. On the other hand, we also provide examples of problems that are as hard in $W$-Stream as in Stream for a given space restriction (regardless of the number of passes). We obtain this result by adapting to $W$-Stream classical communication-complexity arguments used for proving lower bounds in Stream. This kind of arguments cannot instead be applied to StreamSort.

\subsection{Breaking the space wall}

From a space complexity perspective, intermediate streams can be thought of as part of the algorithm's working memory. It is therefore conceivable that one should be able to solve problems in $W$-Stream with a space restriction that would make them unsolvable in Stream. Consider, for instance, the following Parenthesis Language Recognition problem (PLR):

Let $L$ be the context-free parenthesis language $[\mathrm{S} \rightarrow()|(\mathrm{S})|(\mathrm{SS})]$. Let $x$ be a string of $n$ symbols in $\{()$,$\} represented as a data stream. Find out$ if $x \in L$.

We first prove that PLR cannot be solved in Stream using less than logarithmic working memory:

Lemma 4.1. $P L R \notin \operatorname{Stream}(p, o(\log n))$, for any number $p$ of passes.

Proof. Since the parenthesis language is a nonregular context-free language, we can use the following result of Alt et al. [Alt et al. 1992]: if $L$ is a nonregular deterministic context-free language and $L \in N S P A C E(s(n))$, then the recognition of $L$ requires space $s(n) \geq c \cdot \log n$ for some constant $c$ and infinitely many $n$. Clearly, this lower bound also applies to Stream algorithms and implies that, independently of the number of passes, PLR cannot be solved using less than logarithmic space.

On the other hand, we can easily solve PLR in $W$-Stream with a constant size working memory, by removing pairs of consecutive matching parentheses from the stream at each pass, until possible, and returning true if the stream gets empty. Hence, $P L R \in W$-Stream $(n, O(1))$. Our first separation result immediately follows from this observation and from Lemma 4.1:

Theorem 4.2. $\operatorname{Stream}(n, O(1)) \subset \mathrm{W}-\operatorname{Stream}(n, O(1))$.

\subsection{Reducing the number of processed items}

The ability to manipulate the data stream makes it possible, at least for some problems, to discard at each pass items that are no longer useful, thus reducing the overall number of items that an algorithm has to process. Consider, as an example, the following FORK problem: 
Let $A$ and $B$ be two vectors of $n$ numbers with $A[1]=B[1]$ and $A[n] \neq$ $B[n]$. Find a "fork" index $i$ such that $A[i]=B[i]$ and $A[i+1] \neq B[i+1]$.

Assume that $A$ and $B$ are given as an input stream of the form

$$
A[1], A[2], \ldots, A[n], B[1], B[2], \ldots, B[n]
$$

with items in $\{1, \ldots, n\}$. The following lower bound on the space $\times$ passes product follows from a communication complexity lower bound on FORK by Grigni and Sipser [Grigni and Sipser 1995]:

Lemma 4.3. FORK in W-Stream (and thus in Stream) requires $p \times s=\Omega\left(\log ^{2} n\right)$.

Lemma 4.3 implies that, if we stick to logarithmic space, then the number of passes of any streaming algorithm solving FORK must be $p=\Omega(\log n)$. Since in Stream we have to process all the items in the input stream at each pass, it follows that the number of processed items of any Stream algorithm must be $\Omega(n \log n)$ when $s=O(\log n)$.

Instead, we can solve FORK in $W$-Stream more efficiently as follows. Consider a simple binary search-like algorithm, recurring upon the following conditions:

(1) if $A[n / 2]=B[n / 2]$, then there must be a fork index in the second half of the vectors;

(2) if $A[n / 2] \neq B[n / 2]$, then there must be a fork index in the first half of the vectors.

At each pass, we can thus halve the size of the intermediate stream, just by not copying the uninteresting half of the input stream. It is easy to see that this algorithm uses $O(\log n)$ space, runs in $O(\log n)$ passes, and processes only $O(n)$ items overall. From the considerations above, we get our separation with respect to the number of processed items:

THEOREM 4.4. FORK can be (optimally) solved in $\mathrm{W}$-Stream with space $s=$ $O(\log n)$ and $O(n)$ processed items. This is impossible to achieve in Stream.

\subsection{Hardness results}

Even if the use of intermediate streams makes $W$-Stream more powerful than Stream for some problems, in this section we show that for other problems W-Stream maintains all of the hardness of classical streaming. In particular, we exemplify problems for which the use of intermediate streams does not help at all, except for possibly simplifying the task of designing streaming algorithms.

Ruhl and Aggarwal et al. [Aggarwal et al. 2004; Ruhl 2003] already observed that, for a small number of passes, intermediate streams do not help much, regardless of the problem considered. Indeed, W-Stream can be simulated in Stream at the price of increasing the size of the working memory by a factor of $p$ : the simulation given in [Aggarwal et al. 2004; Ruhl 2003] proves that $W$-Stream $(p, s) \subseteq \operatorname{Stream}(p, p \cdot s)$, making intermediate streams unuseful when $p$ is small.

In the following we show that there are problems for which using intermediate streams does not help at all, even regardless of the number of passes. As an example, we take the element-distinctness problem (ED), that asks if there are any duplicates 
in a stream of $n$ numbers in $\{1, \ldots, n\}$. We first give a lower bound on the passes $\times$ space product. Although similar arguments already appear in [Henzinger et al. 1999], we provide a complete proof here as an example of how communicationcomplexity lower bounds used in the Stream model can also be used in W-Stream:

THEOREM 4.5. Any W-Stream algorithm for element distinctness requires $p \times$ $s=\Omega(n)$.

Proof. Consider the bit-vector-disjointness problem, in which Alice and Bob have two $n$-bit-vectors $A$ and $B$, respectively, and want to know whether $A \cdot B>0$. This problem can be reduced to ED in the following way. Alice creates a stream containing the indices corresponding to the 1 's in vector $A$, and Bob does the same for vector $B$. Then Alice runs a $W$-Stream algorithm for element-distinctness on her stream, producing an intermediate stream, and when the input stream is over she sends the content of her working memory to Bob. Bob continues to run the same $W$-Stream algorithm starting from the memory image received from Alice, reading from his own input stream and producing his own intermediate stream. When the stream is over, Bob sends his memory image back to Alice, who starts a second pass by taking as input the intermediate stream that she produced at the previous pass. At the end, the streaming algorithm will determine whether all the elements in the two input streams are distinct or not: notice that the elements are distinct if and only if $A \cdot B>0$, which is exactly the solution to bit-vector-disjointness. If, by contradiction, the total working memory used by Alice and Bob has size $o(n / p)$, then the total number of bits sent between Alice and Bob in $p$ passes would be $o(n / p) \cdot p=o(n)$, which would violate the $\Omega(n)$ communication complexity lower bound for bit-vector-disjointness [Kushilevitz and Nisan 1997].

Since there is a folklore Stream algorithm that solves ED with $p=O(n / s)$ passes, the use of intermediate streams is of no help for this problem.

We remark that arguments similar to the proof of Theorem 4.5 can be used to prove additional lower bounds in $W$-Stream. In particular, many classical communicationcomplexity based lower bounds known in Stream can be adapted to W-Stream, as well. This technique yields, for instance, W-Stream lower bounds for graph problems such as undirected connectivity and shortest paths (see Theorem 2.1 in Section 2 and Theorem 3.1 in Section 3).

\section{CONCLUDING REMARKS}

Data stream processing has enjoyed an increasing popularity in the past few years as a computational paradigm for massive data set applications. Motivated by technological factors, such as the availability of inexpensive secondary storage devices with ever increasing capacity and fast sequential access rates, less restrictive streaming models have been recently proposed. One of these models, named W-Stream, augments the classical streaming model with the ability to sequentially write at each pass an intermediate stream that will be taken as input in the next pass. In this model, we have shown algorithms for fundamental graph problems, such as undirected graph connectivity and directed shortest paths (even with multiple sources). Our algorithms are the first to allow effective tradeoffs between the available internal memory and the number of passes they require. Such tradeoffs are 
not known for the more restrictive classical streaming model, in which intermediate temporary streams are not allowed. Results for other graph problems, such as minimum spanning tree, biconnected components, and maximal independent set, can be obtained by adapting classical parallel algorithms to the $W$-Stream model as recently shown in [Demetrescu et al. 2007].

We conclude by observing that one can achieve space/time tradeoffs for simpler specialized variants of some graph problems even in classical read-only streaming. Consider, for instance, the following chain reachability problem: given a directed chain $C$ and any two nodes $u$ and $v$, determine whether there is a path from $u$ to $v$ in $C$. By using a sampling-based technique inspired by the algorithm described in Section 3, it is not difficult to prove that chain reachability can be solved within $p=O\left(n \log ^{2} n / s\right)$ passes when the space restriction is $s$, without using intermediate temporary streams. It remains, however, an interesting open question whether such space/passes tradeoffs can be achieved in classical streaming for more general and natural graph problems.

Acknowledgments. We wish to thank the anonymous reviewers of the conference version of this paper for many useful comments.

\section{REFERENCES}

Abello, J., Buchsbaum, A., And Westbrook, J. 2002. A functional approach to external graph algorithms. Algorithmica 32, 3, 437-458.

Aggarwal, G., Datar, M., Rajagopalan, S., And Ruhl, M. 2004. On the streaming model augmented with a sorting primitive. In Proceedings of the 45th Annual IEEE Symposium on Foundations of Computer Science (FOCS).

Alon, N., Matias, Y., And Szegedy, M. 1999. The space complexity of approximating the frequency moments. Journal of Computer and System Sciences 58, 1, 137-147.

Alt, H., Geffert, V., And Mehlhorn, K. 1992. A lower bound for the nondeterministic space complexity of context free recognition. Information Processing Letters 42, 25-27.

Babcock, B., Babu, S., Datar, M., Motwani, R., And Widom, J. 2002. Models and issues in data stream systems. In Proceedings of the 21st ACM Symposium on Principles of Database Systems (PODS). 1-16.

Bar-Yossef, Z., Kumar, R., And Sivakumar, D. 2002. Reductions in streaming algorithms, with an application to counting triangles in graphs. In Proceedings of the thirteenth annual ACM-SIAM symposium on Discrete algorithms (SODA 2002), San Francisco, California, pp. 623-632.

Beame, P., Jayram, T., And Rudra, A. 2007. Lower bounds for randomized read/write stream algorithms. In Proceedings of the 39th ACM Symposium on Theory of Computing (STOC'07). To appear.

Cormen, T., Leiserson, C., Rivest, R., And Stein, C. 2001. Introduction to Algorithms, Second Edition. The MIT Press.

Demetrescu, C., Escoffier, B., Moruz, G., And Ribichini, A. 2007. Adapting parallel algorithms to the W-Stream model, with applications to graph problems. Manuscript.

Feigenbaum, J., Kannan, S., McGregor, A., Suri, S., And Zhang, J. 2004. On graph problems in a semi-streaming model. In Proc. of the International Colloquium on Automata, Languages and Programming (ICALP).

Feigenbaum, J., Kannan, S., McGregor, A., Suri, S., And Zhang, J. 2005. Graph distances in the streaming model: the value of space. In Proceedings of the 16th ACM/SIAM Symposium on Discrete Algorithms (SODA). 745-754.

Feigenbaum, J., Kannan, S., Strauss, M., and Viswanathan, M. 2002. An approximate $L^{1}$ difference algorithm for massive data streams. SIAM Journal on Computing 32, 1, 131-151. 
Gilbert, A., Guha, S., Indyk, P., Kotidis, Y., Muthukrishnan, S., and Strauss, M. 2002. Fast, small-space algorithms for approximate histogram maintenance. In Proceedings of the 34th ACM Symposium on Theory of Computing (STOC). 389-398.

Gilbert, A., Kotidis, Y., Muthukrishnan, S., and Strauss, M. 2001. Quicksand: Quick summary and analysis of network data. Tech. rep., DIMACS Technical Report 2001-43.

Golab, L. And Ozsu, M. 2003. Data stream management issues a survey. Tech. rep., School of Computer Science, University of Waterloo, TR CS-2003-08.

Greene, D. And Knuth, D. 1982. Mathematics for the analysis of algorithms. Birkhäuser.

Grigni, M. AND SiPSER, M. 1995. Monotone separation of logarithmic space from logarithmic depth. Journal of Computer and System Sciences 50, 433-437.

Grohe, M., Hernich, A., And Schweikardt, N. 2006. Randomized computations on large data sets: tight lower bounds. In Proc. 25th ACM Symposium on Principles of Database Systems (PODS '06). ACM Press, 243-252.

Grohe, M., Koch, C., and Schweikardt, N. 2005. Tight lower bounds for query processing on streaming and external memory data. In Proc. 32nd Int. Colloquium on Automata, Languages and Programming (ICALP'05). Lecture Notes in Computer Science, vol. 3580. 1076-1088.

Grohe, M. AND SchweIKARDt, N. 2005. Lower bounds for sorting with few random accesses to external memory. In Proc. 24th ACM Symposium on Principles of Database Systems (PODS '05). ACM Press, 238-249.

Henzinger, M. And King, V. 1995. Fully dynamic biconnectivity and transitive closure. In Proc. 36th IEEE Symposium on Foundations of Computer Science (FOCS'95). 664-672.

Henzinger, M., Raghavan, P., And Rajagopalan, S. 1999. Computing on data streams. In "External Memory algorithms", DIMACS series in Discrete Mathematics and Theoretical Computer Science 50, 107-118.

Kushilevitz, E. And Nisan, N. 1997. Communication Complexity. Cambridge Univ. Press.

McGregor, A. 2005. Finding graph matchings in data streams. In Proc. 8th Int. Workshop on Approximation Algorithms for Combinatorial Optimization Problems (APPROX'05). 170-181.

Munro, I. and Paterson, M. 1980. Selection and sorting with limited storage. Theoretical Computer Science 12, 315-323.

Muthukrishnan, S. 2003. Data streams: algorithms and applications. Tech. rep. Available at http://athos .rutgers.edu/ muthu/stream-1-1.ps.

Ruhl, M. 2003. Efficient algorithms for new computational models. Ph.D. thesis, Dept. of Electrical Engineering and Computer Science, MIT.

Sullivan, M. And Heybey, A. 1998. Tribeca: A system for managing large databases of network traffic. In Proceedings USENIX Annual Technical Conference.

Ullman, J. And YANNAKAKIS, M. 1991. High-probability parallel transitive-closure algorithms. SIAM Journal on Computing 20, 1, 100-125.

Vitter, J. 2001. External memory algorithms and data structures: Dealing with massive data. ACM Computing Surveys 33, 2, 209-271. 